\title{
Effect of Topical Versus Sub-conjunctival Anaesthesia during Administration of Intravitreal Bevacizumab Injection
}

\author{
Amna Rizwan ${ }^{1}$, Rabeeah Zafar ${ }^{2}$, Asfandyar Asghar $^{3}$, Naila Obaid ${ }^{4}$, B. A. Naeem ${ }^{5}$ \\ Department of Ophthalmology, ${ }^{1}$ King Edward Medical University, Mayo Hospital, Lahore \\ ${ }^{2}$ Al-Shifa Trust Eye Hospital, ${ }^{3-5}$ Fauji Foundation Hospital, Rawalpindi
}

\begin{abstract}
Purpose: To compare the anesthetic effect of topical proparacaine hydrochloride $0.5 \%$ with sub-conjunctival lidocaine $2 \%$ for intravitreal injection of Bevacizumab.
\end{abstract}

Study Design: Quasi experimental study.

Place and Duration of Study: Department of Ophthalmology Fauji Foundation Hospital, Rawalpindi, from July 2017 to January 2018.

Methods: Sixty 60 patients who needed intravitreal Bevacizumab were included in the study after approval from the ethical review board. Exclusion criteria were patients with conditions that could affect pain sensation, acute ocular inflammation, history of intravitreal injection, using systemic analgesic/sedatives, uncooperative patients and unable to understand the pain scale. Written informed consent was taken. Name, age, gender, diagnosis, previous intravitreal injection, hospital registration number, address and contact numbers were noted. Patients were briefed about the visual analogue scale. Patients were divided into two groups. Group A received proparacaine drops and group B was given subconjunctival lidocaine injections before intravitreal bevacizumab.

Results: The mean age of patients in this study was $60.38 \pm 10.55$ years. There were $03(5.0 \%)$ males and 57 $(95.0 \%)$ females. Majority of the patients $(30 \%)$ presented with choroidal neovascularization followed by diabetic maculopathy, vitreous hemorrhage, central retinal vein occlusion and proliferative diabetic retinopathy. Mean pain score among both the groups was $3.67 \pm 1.97$ (topical group) and $1.70 \pm 1.51$ (sub-conjunctival group) respectively which was statistically significant ( $p$-value 0.000$)$. Among age, diabetes, duration of diabetes and hypertension, only hypertension had moderate correlation with the pain score (correlation coefficient $=0.316$, $p$ values $=0.017$ )

Conclusion: Sub-conjunctival anaesthesia results in less pain during intravitreal drug administration as compared to the topical anaesthesia.

Key Words: Intravitreal injections, Bevacizumab, Intravitreal Anti-VEGF, Anesthesia.

How to Cite this Article: Rizwan A, Zafar R, Asghar A, Obaid N, Naeem BA. Effectiveness of Topical Versus Subconjunctival Anaesthesia during Administration of Intravitreal Bevacizumab Injection. Pak J Ophthalmol. 2021, 37 (4): 356-359.

Doi: $10.36351 /$ pjo.v37i4.1249

Correspondence: Amna Rizwan

King Edward Medical University

Mayo Hospital Lahore

Email:aamnarizwan@hotmail.com

Received: April 3, 2021

Accepted: August 3, 2021

\section{INTRODUCTION}

Intravitreal injections are a standard and effective method for the treatment of posterior segment diseases like endophthalmitis, intraocular inflammation, neovascular glaucoma, retinopathy of prematurity, etc. ${ }^{1,2}$ Recently, use of intravitreal injection has seen 
an upsurge with the maturation of anti-vascular endothelial growth factor (anti-VEGF) drugs for the treatment of age related macular degeneration, diabetic macular edema and macular edema following central or branch retinal vein occlusion. ${ }^{3,4,5}$

Bevacizumab, VEGF-A inhibitor, is a recombinant humanized monoclonal antibody that is typically used for the treatment of colorectal cancer. ${ }^{6,7}$ It is used 'off label' as intravitreal injection as it is cost effective and similar in efficacy to other agents. Side effects include endophthalmitis, transient rise in intraocular pressure, pain, subconjunctival hemorrhage, rhegmatogenous retinal detachment, macular hole, retinal pigment epithelium tear, lens trauma and wound leak. ${ }^{8,9}$

Intravitreal Anti-VEGF treatment requires several injections typically administered on a monthly basis. ${ }^{10}$ It is, therefore, of considerable interest to identify some favorable anesthetics for this procedure. However, to date there is no agreement on the effectiveness of one anaesthetic over another. ${ }^{11}$ This is partly because there is a genuine disagreement between different studies on the expected pain scores in different anesthetics. The most common modes for administration of anesthetics are eye drops, subconjunctival injections, lidocaine gel and lidocaine soaked cotton tipped swabs. ${ }^{12}$

Kaderli and Avci have demonstrated that there is a statistically significant difference in the post intravitreal pain levels with the subconjunctival injection being strongly favored over topical anesthetics. ${ }^{13}$ In particular, Kaderli and Avci reported a mean pain score of $1.64 \pm 0.67$ with topical anaesthetic as opposed to $0.85 \pm 0.2$ for the subconjunctival group $(p<0.001)$ during intravitreal drug administration. In contrast, Karabus et al. claimed no statistically significant difference in the pain score between topical anaesthetics (mean pain score 1.90) and sub-conjunctival injections (mean pain score 1.71, $p=0.746){ }^{4}$ It is this disagreement in the literature that we address in this study.

We propose to investigate the pain level and focus on subconjunctival injections and topical medications as the two anesthetics of choice. This study is important because so far only topical anesthetic is being used in our hospital. Absence of any clear recommendation for the better anaesthetic also makes this study worthwhile. It is, therefore, imperative to get the anesthetic right for this step.

\section{Purpose}

To compare the anesthetic effect of topical proparacaine hydrochloride $0.5 \%$ with subconjunctival lidocaine $2 \%$ for intravitreal injection of Bevacizumab.

\section{METHODS}

This study was conducted at the Department of Ophthalmology, Fauji Foundation hospital, Rawalpindi from July, 2017 to January, 2018. Ethical committee of hospital approved this study. Sampling Technique was non-probability consecutive sampling. Inclusion criteria was age $20-70$ years, both gender and all patients presenting to Ophthalmology Department of the hospital with diseases that required intravitreal bevacizumab injection. Exclusion criteria were patients with conditions that could affect pain sensation, such as acute conjunctivitis, keratitis, stye, acute dacrocystitis and neo-vascular glaucoma, patients who already had received an intravitreal injection, patients using systemic analgesic or sedatives, uncooperative patients and those who were unable to understand the pain scale. Sample size was calculated by WHO calculator which it came out to be 30 in each group. ${ }^{13}$

The nature of study was explained to every subject and a written informed consent was taken. Name, age, gender, diagnosis, previous intravitreal injection, hospital registration number, address and contact numbers were noted. Patients were briefed about the visual analogue scale. Patients were divided into two groups. Group A received proparacaine drops and group B was given subconjunctival lidocaine injections before intravitreal bevacizumab.

The periocular area was cleaned with $10 \%$ povidone iodine and $5 \%$ povidone iodine was instilled in conjunctival sac 3 minutes before the injection. The patients were divided in two groups based on lottery method; one group had received proparacaine drops and the other was given subconjunctival lidocaine injections. A sterile lid speculum was applied and intravitreal injection was given infero-temporally $3.5 \mathrm{~mm}$ away from the limbus in pseudophakic and 4 $\mathrm{mm}$ away from the limbus in phakic eyes. After withdrawing the needle, pressure was applied with a sterilized cotton-tipped applicator to minimize vitreal reflux and sub-conjunctival hemorrhage. Patients were asked about their pain sensation immediately after the injection. The pain score was noted on the visual 
analog pain scale aided by the Wong-Baker faces pain scale. The visual analog pain scale classifies pain on a continuous basis on a scale from 0 to 10 , with 10 signifying the worst imaginable pain. The patients were shown the scale on a chart of A3 paper size so as to identify the pain score with a high sensitivity.

To eliminate bias, anaesthetic and intravitreal injections were given by the same team and investigation of pain was done by the staff nurse who had not known the type of anaesthetic used. Data was entered and analyzed in SPSS version 22.0.

\section{RESULTS}

The mean age for patients in the study was $60.38 \pm$ 10.55 years. There were $03(5.0 \%)$ males and 57 $(95.0 \%)$ females. Female predominance was seen in both groups which could be because of the fact that at this facility, families of ex-servicemen were entitled for treatment.

Frequency and percentage of diagnosis of patients was assessed in the study. Majority of the patients presented with choroidal neovascularization (CNV) $30 \%$, diabetic maculopathy $25 \%$, vitreous hemorrhage $16.7 \%$, central retinal vein occlusion (CRVO) $15 \%$ and proliferative diabetic retinopathy (PDR) $13.3 \%$.

In Table 1 we show the demographic attributes of the two groups. We ran the chi-square test of homogeneity on this data to show that there was no statistically significant difference in the demographic distribution of the two groups from the point of view of hypertension $(p=0.781)$, diabetes $(0.791)$ and the duration of diabetes $(p=0.284)$. While not shown in the table, of the three males, one was assigned to group A and the other two to group B. Hence, the two groups represent homogenous samples.

The objective of the study was to compare topical drops (proparacaine hydrochloride $0.5 \%$ ) and subconjunctival injection (lidocaine 2\%) as anaesthetics for the purpose of intravitreal drug administration. In the study, mean pain score among the two groups was $3.67 \pm 1.97$ and $1.70 \pm 1.51$, respectively. Independent sample t-test was used to compare mean pain score between the two groups which was found to be statistically significant ( $\mathrm{p}$-value 0.000).
Table 1: Demographic attributes of patients included in the study.

\begin{tabular}{lrrrrrrr}
\hline & \multicolumn{4}{c}{ Anesthesia } & & & P \\
& Topical & \multicolumn{2}{c}{$\begin{array}{c}\text { Sub- } \\
\text { Conjunctival }\end{array}$} & Total & & value \\
& $\mathbf{N}$ & $\%$ & $\mathbf{N}$ & $\mathbf{\%}$ & $\mathbf{N}$ & $\%$ & \\
\hline Hypertension & 30 & 100 & 30 & 100 & 60 & 100 & \\
Yes & 21 & 70 & 20 & 66.7 & 41 & 68.3 & 0.781 \\
No & 9 & 30 & 10 & 33.3 & 19 & 31.7 & \\
& & & & & & & \\
Diabetes & 30 & 100 & 30 & 100 & 60 & 100 & \\
Yes & 18 & 60 & 19 & 63.3 & 37 & 61.7 & 0.791 \\
No & 12 & 40 & 11 & 36.7 & 23 & 38.3 & \\
& & & & & & & \\
$\begin{array}{l}\text { Duration of } \\
\text { diabetes }\end{array}$ & 30 & 100 & 30 & 100 & 60 & 100 & \\
$>$ 10 years & 17 & 56.7 & 21 & 70 & 38 & 63.3 & 0.284 \\
$<$ 10 years & 13 & 43.3 & 9 & 30 & 22 & 36.7 & \\
\hline
\end{tabular}

Applying Pearson's coefficient of correlation of pain score with the age, as well as the dichotomous categorical variables of diabetes, duration of diabetes and hypertension, only hypertension had a statistically significant moderate correlation with the pain score (correlation coefficient $=0.316, \mathrm{p}$ values $=0.017$ )

In Table 2, comparison between the two groups regarding complications of administering the intravitreal injection are shown.

Table 2: Complications of intravitreal injections in the two groups.

\begin{tabular}{llll}
\hline & Complications & Group A & Group B \\
\hline 1 & Hyperemia & $7(23 \%)$ & $9(30 \%)$ \\
2 & Sub-conjunctival hemorrhage & $5(16.6 \%)$ & $11(36.6 \%)$ \\
3 & Chemosis & $2(6.67 \%)$ & $16(53.3 \%)$ \\
4 & Keratitis & Nil & Nil \\
5 & Raised IOP & Nil & Nil \\
6 & Uveitis & Nil & Nil \\
7 & Endophthalmitis & Nil & Nil \\
\hline
\end{tabular}

\section{DISCUSSION}

Intravitreal injections were first described by Deutschmann and Ohm who used rabbit vitreous and air to repair retinal detachments in humans. ${ }^{14}$ Nowadays, antivirals, antibiotics, anti-inflammatory and vascular endothelial growth factor (anti-VEGF) are given intravitreally as a standard ophthalmic practice.

Intravitreal injections are now a part of the standard treatment for retinal diseases. It is, therefore, important to make the experience of intravitreal drug 
administration as pain-free for the patient as possible. A study by the American Society of Retina Specialists reported in 2010 that there was no clear preference for use of anaesthetic during intravitreal injection administration. It was found that $25.44 \%$ specialists preferred topical anaesthetic drops, $25.15 \%$ used a topical gel, $26.33 \%$ used pledget in combination with drops, while $22.19 \%$ used subconjunctival injections. ${ }^{4}$

The mean pain score of our study is $2.68 \pm 2.00$ for both groups which is consistent with the mean pain score of $2.14 \pm 1.90$ reported by Kaderli et al. ${ }^{13}$ In our study, mean age (years) in the study was $60.38 \pm 10$. Similarly, for Kaderli et al, ${ }^{13}$ mean age in years was 59 \pm 14.5 .

In our study, mean pain score of topical and subconjunctival groups was $3.67 \pm 1.97$ and $1.70 \pm$ 1.51 respectively. The difference is statistically significant $(\mathrm{p}=0.000)$. Likewise, Kaderli and Avcireport reported mean pain score of $1.64 \pm 0.67$ with topical anaesthetic as opposed to $0.85 \pm 0.2$ for the sub-conjunctival group $(p<0.001)$ during intravitreal drug administration. ${ }^{13}$ Similarly, Andrade et al, compared the effectiveness of topical proparacaine, subconjunctival lidocaine and $2 \%$ lidocaine gel. They reported that $86.2 \%$ patients who received sub-conjunctival anaesthetic rated the overall procedure of receiving an intravitreal injection as very good or excellent $(37.9 \%)$. No one receiving topical drops reported the procedure as being excellent and only $19.2 \%$ reported it as being very good. Patients in topical drops group had the worst mean pain scores during the injection. They concluded subconjunctival lidocaine was most effective in preventing pain. Lidocaine gel was effective but it had high incidence of keratitis. They also studied the eye movement while administering the intravitreal injection and concluded that sub-conjunctival group had the least eye movement consistent with a low mean pain score. Whereas, chemosis was high in subconjunctival group, the overall conclusion of the superiority of using subconjunctival anaesthetic is quite consistent with our findings. ${ }^{15}$

In our study, chemosis was more in subconjunctival group which is consistent with results of Andarde et al. ${ }^{15}$ Kozak et al reported a higher rate of chemosis in the subconjunctival group. ${ }^{16}$ While Andarde et $\mathrm{al}^{15}$ advocated the use of subconjunctival anaesthetic as compared to lidocaine gel, Kozak et al ${ }^{16}$ had recommended the use of gel based on higher rate of chemosis.
Karabus et al found higher incidence of subconjunctival hemorrhage in patients of sub conjunctival group which is consistent with our study. ${ }^{4}$ However, it is to be noted that whereas $36.6 \%$ patients reported subconjunctival hemorrhage as compared to $16.6 \%$ patients in topical group, the hemorrhage was not troublesome to the patient. In our experience, chemosis and subconjunctival hemorrhage were not very bothersome to the patients as compared to the pain.

Blaha et al found no statistically significant difference in pain score while comparing four different anaesthetic methods (proparacaine, tetracaine, lidocaine pledget and subconjunctival injection of lidocaine). ${ }^{17}$ This, of course, is in contradiction to our study as well as that of Kaderli et al. ${ }^{13}$

Rifkin and Schaal observed factors that could influence pain during in-office intravitreal drug administration. ${ }^{10}$ They observed factors (improved vision from previous injection, female gender, age greater than 65 years and number of injection) that could influence pain. Pain decreased with each subsequent injection. We therefore excluded such patients (those that had received intravitreal drugs in the past).

Cohen et al concluded that most patients preferred sub conjunctival anesthesia to topical anesthesia for intravitreal injections. ${ }^{18}$ They suggested to give ample time after subconjunctival anaesthetic and based their recommendations on patients' preference. According to them $88 \%$ patients preferred subconjunctival anesthesia and $12 \%$ preferred topical anesthesia for on-going treatments. ${ }^{18}$

A survey of Canadian retina specialists showed that at least a quarter of them routinely used subconjunctival anaesthetics and up to half of them infrequently used the same. ${ }^{19,20}$

Complications reported in the literature of intravitreal injections include raised intraocular pressure, uveitis and endophthalmitis. No patient in our study reported these complications.

The drawbacks in our study are small sample size and short term follow-up. These should be addressed in a more detailed analysis.

\section{CONCLUSION}

Subconjunctival anaesthesia results in less pain during intravitreal drug administration compared with topical anaesthesia. 


\section{Ethical Approval}

The study was approved by the Institutional review board/Ethical review board. (469-RC/FFH/RWP)

\section{Conflict of Interest}

Authors declared no conflict of interest.

\section{REFERENCES}

1. Haas P, Falkner-Radler C, Wimpissinger B, Malina M, Binder S. Needle size in intravitreal injections pain evaluation of a randomized clinical trial. Acta Ophthalmol. 2016; 94 (2): 198-202.

2. Shikari H, Samant PM. Intravitreal injections: A review of pharmacological agents and techniques. J Clin Ophthalmol. Res. 2016; 4: 51-59.

3. Shimura M, Kitano S, Muramatsu D, Fukushima H, Takamura Y, Matsumoto M, et al. Real-world management of treatment-naïve diabetic macular oedema in Japan: two-year visual outcomes with and without anti-VEGF therapy in the STREAT-DME study. Br J Ophthalmol. 2020; 104 (9): 1209-1215.

Doi: 10.1136/bjophthalmol-2019-315199.

4. Karabus VL, Ozkan B, Kocer CA, Altintas O, Pirhan D, Yuksel N. Comparison of two anesthetic methods for intravitreal Ozurdex injection. J Ophthalmol. 2015; 2015: 861535.

5. Fukami M, Iwase T, Yamamoto K, Kaneko H, Yasuda S, Terasaki H. Changes in Retinal Microcirculation After Intravitreal Ranibizumab Injection in Eyes with Macular Edema Secondary to Branch Retinal Vein Occlusion. Invest Ophthalmol Vis Sci. 2017; 58 (2): 1246-1255.

Doi: https://doi.org/10.1167/iovs.16-21115.

6. Ferrara $\mathbf{N}$. Humanization of an Anti-VEGF Monoclonal Antibody for the Therapy of Solid Tumors and Other Disorders. Cancer Res. 2016; 76 (17): 49134915. Doi: 10.1158/0008-5472.CAN-16-1973.

7. Franchi M, Barni S, Tagliabue G, Ricci P, Mazzucco W, Tumino R, et al. Effectiveness of First-Line Bevacizumab in Metastatic Colorectal Cancer: The Observational Cohort Study GRETA. Oncologist, 2019; 24 (3): 358-365.

Doi: 10.1634/theoncologist.2017-0314.

8. Pradhan E, Duwal S, Bajimaya S, Thapa R, Sharma S, Manandhar A. Acute Endophthalmitis after Intravitreal Bevacizumab Injections at The Tertiary Centre in Nepal. Nepal J Ophthalmol. 2018; 10 (19): 107-110. Doi: 10.3126/nepjoph. v10i1.21727.

9. Park J, Lee M. Short-term effects and safety of an acute increase of intraocular pressure after intravitreal bevacizumab injection on corneal endothelial cells. BMC Ophthalmol. 2018; 18 (1): 17.

Doi: 10.1186/s12886-018-0682-9.
10. Rifkin L, Schaal S. Factors affecting patients' pain intensity during in office intravitreal injection procedure. Retina, 2012; 32: 696-700.

11. Azmeh AM. Ocular and Systemic Vascular Adverse Events Following Intravitreal Bevacizumab Injection. J Ophthalmol Vis Sci. 2016; 1: 1004.

12. Yau GL, Jackman CS, Hooper PL, Sheidow TG. Intravitreal injection anesthesia-comparison of different topical agents: a prospective randomized controlled trial. Am J Ophthalmol. 2011; 151: 333-337.

13. Kaderli B, Avci R. Comparison of topical and subconjunctival anesthesia in intravitreal injection administrations. Eur J Ophthalmol. 2006; 16: 718-721.

14. Shikari H, Samant PM. Intravitreal injections: A review of pharmacological agents and techniques. J Clin Ophthalmol. 2016; 4: 51-59.

15. Andrade GC, Carvalho ACM. Comparison of 3 different anesthetic approaches for intravitreal injections: a prospective randomized trial. Arq Bras Oftalmol. 2015; 78: 27-31.

16. Kozak I, Cheng L, Freeman WR. Lidocaine gel anesthesia for intravitreal drug administration. Retina, 2005; 25 (8): 994-998.

17. Blaha GR, Tilton EP, Barouch FC, Marx JL. Randomized trial of anesthetic methods for intravitreal injections. Retina 2011; 31 (3): 535-539.

18. Cohen SM, Billiris-Findlay K, Eichenbaum DA, Pautler SE. Topical lidocaine gel with and without subconjunctival lidocaine injection for intravitreal injection: a within-patient study. Ophthalmic Surg Lasers Imaging Retina, 2014; 45 (4): 306-310.

19. Xing L, Dorrepaal SJ, Gale J. Survey of intravitreal injection techniques and treatment protocols among retina specialists in Canada. Can J Ophthalmol. 2014; 49 (3): 261-266. Doi: 10.1016/j.jcjo.2014.03.009.

20. Han J, Rinella NT, Chao DL. Anesthesia for Intravitreal Injection: A Systematic Review. Clin Ophthalmol. 2020; 14: 543-550.

Doi:10.2147/OPTH.S223530.

\section{Authors' Designation and Contribution}

Amna Rizwan; Vitreoretina Fellow: Concepts, Design, Literature search, Data acquisition, Data analysis, Statistical analysis, Manuscript preparation.

Rabeeah Zafar; Registrar: Data acquisition, Manuscript editing, Manuscript review.

Asfandyar Asghar; Professor: Manuscript editing, Manuscript review.

Naila Obaid; Assistant Professor: Literature search, Data acquisition. Manuscript review

B. A. Naeem; Professor and HOD: Manuscript review. 